Proceedings

\title{
Comparative Evaluation of the Total Antioxidant Capacities of Plant Polyphenols in Different Natural Sources $^{+}$
}

\author{
María del Carmen Villegas-Aguilar 1,2,*, María de la Luz Cádiz-Gurrea 1,2, David Arráez-Román 1,2 \\ and Antonio Segura-Carretero ${ }^{1,2}$ \\ 1 Department of Analytical Chemistry, University of Granada, Granada, Spain; \\ mluzcadiz@ugr.es (M.d.l.L.C.-G.); darraez@ugr.es (D.A.-R.); ansegura@ugr.es (A.S.-C.) \\ 2 Research and Development of Functional Food Centre (CIDAF), Granada, Spain \\ * Correspondence: marivillegas@ugr.es \\ + Presented at the 1st International e-Conference on Antioxidants in Health and Disease, 01-15 December \\ 2020; Available online: https://cahd2020.sciforum.net/.
}

Published: 30 November 2020

\begin{abstract}
Phenolic compounds during the last decades have been the object of study by the scientific community due to their high and diverse bioactive potential, being their antioxidant capacity one of the most studied since due to their structure they have a high potential to act as effective compounds against oxidative stress. Due to their great structural diversity, these compounds have been classified into at least 10 different classes based on their basic chemical structure. The aim of this work was to carry out the characterization of the main phenolic content and the determination of the antioxidant capacity of 4 plant matrices. Firstly, Lippia citriodora, Hibiscus sabdariffa, Olea europaea and Silybum marianum were chosen as natural sources due to their high content of phenolic compounds. After that, a qualitative characterization of the phenolic profile from the selected plant extracts was performed using a high-performance liquid chromatography coupled to mass spectrometry was carried out. Finally, the determination of the total content of polyphenols was carried out using the Folin-Ciocalteu method and the antioxidant capacity using the electron transfer methods, FRAP and TEAC, and hydrogen donation, ORAC. After the chromatographic analysis of the phenolic profile of each matrix, it was observed that there are different major phenolic compound families for each plant matrix. Regarding the antioxidant capacity, L. citriodora showed better results for the FRAP assay, while for the TEAC and ORAC assays the highest values were for S. marianum.
\end{abstract}

Keywords: phenolic compounds; antioxidants; HPLC-MS

\section{Introduction}

Phenolic compounds present in plants are metabolites that have been shown to have a strong antioxidant capacity. Despite the fact that plants synthesize them mainly for their own defense against oxidative stress, these compounds retain the ability to act as ex-plant antioxidants and, therefore, they contribute greatly to the dietary and pharmaceutical properties of plant-food [1]. These compounds have a wide range of structures and functions, generally, they possess an aromatic ring with one or more hydroxyl substituents and have been classified into at least 10 different classes based on their basic chemical structure [1]. However, there is no universal method for the measurement of antioxidant capacity, since these can measure different mechanisms of action, such 
The 1st International Electronic Conference on Antioxidants in Health and Disease, 1-15 December 2020 as the transfer of electrons or the transfer of hydrogen atoms. In this sense, the selection of different assays to evaluate antioxidant activity would allow us to obtain a complete prediction of this bioactive potential [1,2]. The aim of this work has been to know the main families of phenolic compounds and to measure the antioxidant capacity through different methods of four selected plant matrices in order to obtain a complete prediction of their antioxidant potential and the relationship of this bioactivity with the more abundant phenolic families in these.

\section{Material and Methods}

\subsection{Chemicals and Reagents}

All reagents used in this work were analytical grade and used without changes. The analytical procedures were performed using water purified by a Milli-Q system from Millipore (Bedford, MA, USA). LC-MS grade methanol and acetic acid were purchased from Fisher Chemicals (Waltham, MA, USA) and Sigma-Aldrich (Steinheim, Germany), respectively.

To measure the antioxidant capacity and total phenolic content, the following reagents were provided from the indicated suppliers: AAPH (2,2'-azobis-2-methyl-propanimidamide, dihydrochloride), ABTS [2,2'-azinobis (3-ethylbenzothiazoline-6-sulphonate)], ferric sulfate, FolinCiocalteu reagent, potassium persulfate, TPTZ (2,4,6-tripyridyl-S-triazine), Trolox (6-hydroxy-2,5,7,8tetramethylchroman-2-carboxylic acid) from Sigma-Aldrich (St. Louis, MO, USA). Gallic acid, sodium acetate, ferric chloride, hydrochloric acid, trihydrated sodium acetate, dehydrated sodium phosphate and sodium carbonate provided from Panreac (Barcelona, Spain).

\subsection{Sample Preparation}

L. citriodora leaves, H. sabdariffa calyces, O. europaea leaves and S. marianum fruits extracts were provided by Natac (Madrid, Spain). The dry extracts were stored in darkness and kept at room temperature until their use. Prior to use, the dry extracts were reconstituted in the same extraction solvent mixture to a concentration of $5 \mathrm{mg} / \mathrm{mL}$ and filtered with single-use syringe filters $(0.20 \mu \mathrm{m}$ pore size).

\subsection{HPLC-ESI-TOF-MS Analysis}

An RRLC 1200 system was used to analyze the extracts (Agilent Technologies, Palo Alto, CA, USA). This analytical platform was equipped with a vacuum degasser, an automated sampler, a binary solvent delivery system and a UV-Vis detector. The RRLC system was coupled to a Bruker micrOTOF mass spectrometer (Bruker Daltonik, Bremen, Germany) using an electrospray interface (ESI) (model G1607 from Agilent Technologies, Palo Alto, CA, USA). Phytochemical separation was accomplished using a $150 \mathrm{~mm} \times 4.6 \mathrm{~mm}$ id, $1.8 \mu \mathrm{m}$ particle diameter Zorbax Eclipse Plus C18 column (Agilent Technologies, Palo Alto, CA, USA). The eluents were water with $0.5 \%$ acetic acid (eluent A) and methanol (eluent B). Briefly, the multistep gradient used to separate the phytochemicals in L. citriodora was $0 \mathrm{~min}, 0 \% \mathrm{~B} ; 15 \mathrm{~min}, 60 \% \mathrm{~B} ; 33 \mathrm{~min}, 100 \% \mathrm{~B} ; 38 \mathrm{~min}, 100 \% \mathrm{~B} ; 38 \mathrm{~min}, 100 \% \mathrm{~B} ; 46 \mathrm{~min}, 0 \% \mathrm{~B} ; 55 \mathrm{~min}$, $0 \% \mathrm{~B}$ min. For $H$. sabdariffa the gradient was $0 \mathrm{~min}, 0 \% \mathrm{~B} ; 5 \mathrm{~min}, 25 \% \mathrm{~B} ; 20 \mathrm{middccn}, 40 \% \mathrm{~B} ; 30 \mathrm{~min}, 60 \%$ B; $38 \mathrm{~min}, 100 \% \mathrm{~B} ; 46 \mathrm{~min}, 0 \% \mathrm{~B} ; 55 \mathrm{~min}, 0 \% \mathrm{~B}$; for O. europaea $0 \mathrm{~min}, 0 \% \mathrm{~B} ; 5 \mathrm{~min}, 30 \% \mathrm{~B} ; 20 \mathrm{~min}, 60 \%$ $\mathrm{B} ; 33 \mathrm{~min}, 100 \% \mathrm{~B} ; 38 \mathrm{~min}, 100 \% \mathrm{~B} ; 46 \mathrm{~min}, 0 \% \mathrm{~B} ; 55 \mathrm{~min}, 0 \% \mathrm{~B}$ and for $\mathrm{S}$. marianum $0 \mathrm{~min}, 0 \% \mathrm{~B} ; 7 \mathrm{~min}$, $60 \% \mathrm{~B} ; 25 \mathrm{~min}, 100 \% \mathrm{~B} ; 30 \mathrm{~min}, 100 \% \mathrm{~B} ; 36 \mathrm{~min}, 0 \% \mathrm{~B} ; 45 \mathrm{~min}, 0 \% \mathrm{~B}$. Finally, a conditioning cycle (10 $\mathrm{min}$ ) was applied with initial conditions before each injection. The injection volume was $10 \mu \mathrm{L}$ at room temperature. The flow rate was $0.3 \mathrm{~mL} / \mathrm{min}$. The effluent from the HPLC system was introduced into

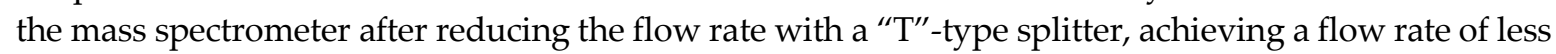
than $0.2 \mathrm{~mL} / \mathrm{min}$ and ensuring correct ionization by ESI. All MS assays were developed in negative ion mode and considering a mass range from 50 to $1000 \mathrm{~m} / \mathrm{z}$. The identification was accomplished with the use of Data Analysis 4.0 software (Bruker Daltonics, Billerica, MA, USA), whose sophisticated CHNO algorithm supported a good identification. Measurements were made in triplicate. 
The 1st International Electronic Conference on Antioxidants in Health and Disease, 1-15 December 2020

2.4. Total Phenolic Content by Folin Ciocalteu

The total phenolic content was measured by Folin-Ciocalteu method with some modifications [3]. Dry extracts were dissolved in ethanol:water (80:20) (different concentrations were tested). The absorbance measurement was carried out on a Synergy Mx Monochromator-Based Multi-Mode Micro plate reader (Bio-Tek Instruments Inc., Winooski, VT, USA) using 96-well polystyrene microplates. Phenol content was calculated based on the calibration curves of Gallic Acid and expressed as mg Gallic acid equivalents/g of dry extract. The experiments were made in triplicate.

\subsection{Antioxidant Activity Assays}

The antioxidant activity of the dry extracts were evaluate using two methods based on singleelectron transfer (SET): FRAP and TEAC assays and the oxygen radical absorbance capacity (ORAC) method as a hydrogen-atom transfer (HAT) based method. To carry out these measurements, the same plate reader mentioned above was used.

\subsubsection{Ferric Reducing Antioxidant Power}

The FRAP assay was carried out following the method described by Benzie and Strain (1996) [4]. In this method, FRAP values were calculated by measuring absorbance at $593 \mathrm{~nm}$ in a microplate reader. A standard curve of $\mathrm{FeSO}_{4} \cdot 7 \mathrm{H}_{2} \mathrm{O}$ was evaluated and finally the results were expressed in mmol of mmol $\mathrm{FeSO}_{4}$ equivalents/g of dry extract. The experiments were carried out in triplicate.

\subsubsection{Trolox Equivalent Antioxidant Capacity}

The TEAC test originally described by Miller et al. (1993) with some modifications according to Cádiz-Gurrea et al. (2013) [2,5]. TEAC values were calculated using Trolox as a standard and reading absorbance at $734 \mathrm{~nm}$ on a microplate reader. The results were expressed in mmol of Trolox equivalents/g of dry extract. The experiments were carried out in triplicate.

\subsubsection{Oxygen Radical Absorbance Capacity}

The ORAC method developed by Ou et al. (2001) was carried out with the modifications carried out by Cádiz-Gurrea et al. (2013) [2,6]. In this assay where fluorescence is measured, Trolox was used as the reference compound and a regression equation was used between its concentration and the net area of the fluorescence decay curve (area under the curve, AUC) to calculate the Final ORAC values of the expressed samples mmol of Trolox equivalents/g of dry extract. The experiments were carried out in triplicate.

\section{Results and Discussion}

\subsection{Characterization by HPLC-ESI-TOF-MS}

In the identification of L. citriodora by HPLC-ESI-TOF-MS, it was observed that organic acids were present, such as gluconic acid, flavonoids like chrysoeriol-diglucuronide, iridoids and secoiridoids such as loganic acid and shanziside, and phenilpropanoids as verbasoside and verbascoside, the latter being one of the compounds found with the highest concentration. In the case of $\mathrm{H}$. sabdariffa, after the analysis it was observed that organic acids such as hibiscus acid, phenolic acids such as caffeoylquinic acid and flavonoids like quercitine ad myricetin were present. In O. europaea a greater diversity of groups present in its composition were found, such as the group of organic acids like quinic acid, phenolic acids such as mycophenolic acid, simple phenols for instance hydroxytyrosol, flavonoids such as epigallocatechin acid and kaempferol, secoiridoids like oleuropein and phenylpropanoids as acteoside. In relation to the S. marianum plant matrix, the following groups were identified: organic acids such as oxydobenzoic acid, diterpenes with 
The 1st International Electronic Conference on Antioxidants in Health and Disease, 1-15 December 2020 compounds like taxuspin, and flavonoids such as dihydroquercetin and sylibin, the latter being one of the major compounds in the plant matrix.

\subsection{Total Phenolic Content and Antioxidant Activities}

Table 1 shows the total phenolic content measured by the Folin-Ciocalteu method of the five plant extracts under study. As can be seen, the highest content is found in S. marianum with a value of $536.95 \pm 56.94 \mathrm{mg}$ Gallic acid equivalents/g of dry extract, being much higher than the rest of the plant extracts under study. This may be due to the fact that in the case of S. marianum the extract is from its fruit, while for L. citriodora and O. europaea it is made with its leaves and H. sabdariffa with its calyces. In this sense, since it is an extract obtained from the fruit, there may be a high content of sugars, which has been seen to interfere with the Folin-Ciocalteu test [7], making this much higher.

Table 1. In vitro total phenolic content and antioxidant capacity by TEAC, FRAP and ORAC methods of L. citrtiodora, H. sabdariffa, O. europaea and S. marianum extracts.

\begin{tabular}{lcccc}
\hline & Folin-Ciocalteu $^{\mathrm{a}}$ & FRAP $^{\mathrm{b}}$ & TEAC $^{\mathrm{c}}$ & ORAC $^{\mathrm{d}}$ \\
\hline L. citriodora & $293.96 \pm 2.44$ & $2.57 \pm 0.09$ & $1.13 \pm 0.06$ & 5.93 \\
H. sabdariffa & $51.09 \pm 1.41$ & $0.47 \pm 0.05$ & $0.24 \pm 0.04$ & 1.16 \\
O. europaea & $216.25 \pm 28.70$ & $1.67 \pm 0.02$ & $0.84 \pm 0.05$ & 6.01 \\
S. marianum & $536.95 \pm 56.94$ & $1.41 \pm 0.28$ & $1.34 \pm 0.12$ & 11.71 \\
\hline
\end{tabular}

a Expressed in mg Gallic acid equivalents/g of dry extract; ${ }^{b}$ Expressed in mmol $\mathrm{FeSO}_{4}$ equivalents/g of dry extract; ${ }^{c}$ Expressed in mmol Trolox equivalents/g of dry extract; ${ }^{d}$ Expressed in mmol Trolox equivalents/g of dry extract.

In the case of FRAP (Table 1), the plant matrix that has reported the highest values is L. citriodora with $2.57 \pm 0.09 \mathrm{mmol} \mathrm{FeSO}_{4}$ equivalents/g of dry extract while the plant matrix with the lowest results has been $\mathrm{H}$. sabdariffa with $0.47 \pm 0.05 \mathrm{mmol} \mathrm{FeSO} 4$ equivalents/g of dry extract. For the matrices $\mathrm{O}$. europaea and $S$. marianum, the values were intermediate in comparison with the previous ones, being $1.67 \pm 0.02$ and $1.41 \pm 0.28 \mathrm{mmol} \mathrm{FeSO}_{4}$ equivalents/g of dry extract, respectively. The high value obtained in this test for L. citriodora may be due to its high content of verbascoside, a characteristic and abundant phenylproanoid in it, since the positive relationship between the amount of verbascoside in the matrix and the higher capacity in the FRAP test has been confirmed by various studies $[1,8]$.

Despite the fact that both FRAP and TEAC (Table 1) are methods that determine the antioxidant capacity through electron transfer, the results obtained for the different matrices are not the same. In this sense, it is observed that in the case of TEAC, the matrix with the highest result compared to the others has been $S$. marianum with $1.34 \pm 0.12 \mathrm{mmol}$ Trolox equivalents/g of dry extract, followed by L. citriodora with $1.13 \pm 0.06 \mathrm{mmol}$ Trolox equivalents/g of dry extract which, in the case of FRAP, was the one with the greatest iron ion reduction capacity. This agrees with the results obtained by Valentová et al. (2018) in which they observed that sylibin, a flavonoid present in high concentrations in the fruit of $S$. marianum, had a higher antioxidant capacity in the TEAC test compared to the FRAP test, which indicates the better capacity of reduction of ABTS, which is the measure of TEAC, than reduction of iron ion, the method on which the FRAP is based [9].

In relation to the ORAC assay (Table 1), which is based on the hydrogen-atom transfer, a trend similar to what occurs in the TEAC test is observed, since it is observed that it is the S. marianum matrix that has the highest value with $11.71 \mathrm{mmol}$ Trolox equivalents $\mathrm{g}$ of dry extract. However, in this case the value for L. citriodora and O. europaea is very similar, being 5.93 and 6.01 mmol Trolox equivalents/g of dry extract respectively. The results agree with those obtained by Viktorova et al. (2019) as they observed that both the S. marianum extract and its major flavonoids in isolation had a high action capacity in the ORAC test, exerting a better hydrogen-atom transfer capacity than the ABTS reduction capacity by electron transfer [10]. This may suggest that the group of flavonoids 
The 1st International Electronic Conference on Antioxidants in Health and Disease, 1-15 December 2020

present in S. marianum has a better capacity to transfer hydrogen atoms than the majority and characteristic phenylpronaoids in both L. citriodora and O. europaea.

It should be noted that for the H. sabdariffa plant matrix it is shown that both the total phenolic content and the antioxidant activity measured by the different methods used have presented a very low value compared to the rest of the plant matrices under study, which may be due to to the fact that the extraction of phenolic compounds has not been carried out completely, which indicates the importance of the selection of the conditions and the form of extraction of the compounds under study as the first step for a study of biaoactivity of an extract.

\section{Conclusions}

A comprehensive characterization and biological antioxidant properties of selected sources have been obtained. After the chromatographic analysis of the phenolic profile of each matrix, it was observed that in L. citriodora the abundance of the group of phenylpropanoids and iridoids stands out, in H. sabdariffa the groups of anthocyanins and flavonoids, in O. europaea secoiridoids and terpenes while in $S$. marianum the group of flavonoids. Regarding the antioxidant capacity, $L$. citriodora showed better results for the FRAP assay, while for the TEAC and ORAC assays the highest values were for $S$. marianum. In this sense, it is important to note that there is no universal method for measuring antioxidant capacity, so it is convenient to use a set of tests to evaluate antioxidant activity since no single method can give a complete prediction of this bioactive potential.

Acknowledgments: The authors would like to thank the Ministry of Science, Innovation and Universities (RTI2018-096724-B-C22). The author M.d.C.V.-A. gratefully acknowledges the Spanish Ministry of Science, Innovation and Universities for the FPU grant FPU19/01146.

Author Contributions: M.d.1.L.C.-G. and D.A.-R. conceived and designed the experiments; M.d.C.V.-A. performed the experiments; M.d.C.V.-A. and M.d.l.L.C.-G. analyzed the data; A.S.-C. and D.A.-R. contributed reagents/materials/analysis tools; M.d.C.V.-A. wrote the paper.

Conflicts of Interest: The authors declare no conflict of interest.

\section{References}

1. Sánchez-Marzo, N.; Lozano-Sánchez, J.; de la Luz Cádiz-Gurrea, M.; Herranz-López, M.; Micol, V.; SeguraCarretero, A. Relationships between chemical structure and antioxidant activity of isolated phytocompounds from lemon verbena. Antioxidants 2019, 8, 324.

2. de la Luz Cádiz-Gurrea, M.; Fernández-Arroyo, S.; Joven, J.; Segura-Carretero, A. Comprehensive characterization by UHPLC-ESI-Q-TOF-MS from an Eryngium bourgatii extract and their antioxidant and anti-inflammatory activities. Food Res. Int. 2013, 50, 197-204.

3. Cádiz-Gurrea, M.D.L.L.; Borrás-Linares, I.; Lozano-Sánchez, J.; Joven, J.; Fernández-Arroyo, S.; SeguraCarretero, A. Cocoa and grape seed byproducts as a source of antioxidant and anti-inflammatory proanthocyanidins. Int. J. Mol. Sci. 2017, 18, 376.

4. Benzie, I.F.F.; Strain, J.J. The Ferric Reducing Ability of Plasma (FRAP) as a Measure of "Antioxidant Power": The FRAP Assay. Anal. Biochem. 1996, 239, 70-76.

5. Miller, N.J.; Rice-Evans, C.; Davies, M.J.; Gopinathan, V.; Milner, A. A novel method for measuring antioxidant capacity and its application to monitoring the antioxidant status in premature neonates. Clin. Sci. 1993, 84, 407-412.

6. Ou, B.; Hampsch-Woodill, M.; Prior, R.L. Development and Validation of an Improved Oxygen Radical Absorbance Capacity Assay Using Fluorescein as the Fluorescent Probe. J. Agric. Food Chem. 2001, 49, 46194626.

7. Sánchez-Rangel, J.C.; Benavides, J.; Heredia, J.B.; Cisneros-Zevallos, L.; Jacobo-Velázquez, D.A. The FolinCiocalteu assay revisited: Improvement of its specificity for total phenolic content determination. Anal. Methods 2013, 5, 5990-5999. 
The 1st International Electronic Conference on Antioxidants in Health and Disease, 1-15 December 2020

8. Orak, H.H.; Magdalena, K.; Amarowicz, R.; Orak, A.; Penkacik, K. Genotype-Related Differences in the Phenolic Compound Profile and Antioxidant Activity of Extracts from Olive (Olea europaea L.) Leaves. Molecules 2019, 24, 1130.

9. Valentová, K.; Purchartová, K.; Rydlová, L.; Roubalová, L.; Biedermann, D.; Petrásková, L.; Křenková, A.; Pelantová, H.; Holečková-Moravcová, V.; Tesařová, E.; et al. Sulfated metabolites of flavonolignans and 2,3-dehydroflavonolignans: Preparation and properties. Int. J. Mol. Sci. 2018, 19, 2349.

10. Viktorova, J.; Stranska-Zachariasova, M.; Fenclova, M.; Vitek, L.; Hajslova, J.; Kren, V.; Ruml, T. Complex evaluation of antioxidant capacity of milk thistle dietary supplements. Antioxidants 2019, 8, 317.

(C) 2020 by the authors; licensee MDPI, Basel, Switzerland. This article is an open access article distributed under the terms and conditions of the Creative Commons by Attribution (CC-BY) license (http://creativecommons.org/licenses/by/4.0/). 